\title{
PROLOGO
}

\section{INFORME DE LA FIGO SOBRE MONITOREO E INFORMACION DE MORTALIDAD Y MORBILIDAD PERINATALES}

Este informe, resultante del taller del Comité de Mortalidad y Morbilidad Perinatal de Heidelberg, fué presentado al $10^{\circ}$ Congreso Mundial de F.I.G.O., que tuvo lugar en San Francisco, en Octubre de 1982. Este informe recibió total aprobación y apoyo tanto del Comité Ejecutivo, cuanto de la Asamblea General integrada por los delegados de las sociedades miembros.

La gran importancia de los datos recolectados, análisis e informe fué reconocida por la Asamblea General de F.I.G.O., la que aprobó la sugestión de crear un Subcomité del Comité Permanente de F.I.G.O. sobre Mortalidad y Morbilidad Perinatal, con el título de "Estadísticas de Epidemiología y Salud Perinatal". El Dr. P. M. Dunn fué designado su Coordinador.

F. Kubli

Coordinador

Comité Permanente de Mortalidad y Morbilidad Perinatal,

Universitäts - Frauenklinik, Heidelberg, Alemania Occidental.
P. M. Dunn

Coordinador

Comité de Estadísticas

de Epidemiología y

Salud Perinatal.

Southmead Hospital, Bristol, U.K. 


\section{La reunión de trabajo de FIGO sobre Monitoreo e Información en Mortalidad y Morbilidad Perinatal tuvo lugar en la Clínica de Mujeres de la Universidad de Heidelberg, Alemania, en Marzo 19 al 21 de 1982.}

El Coordinador, Prof. F. Kubli, dió la bienvenida a los participantes. El Prof. G. Rooth fué nombrado Vice-Coordinador y el Dr. P. M. Dunn, informante. La reunión de trabajo adoptó un programa flexible de presentaciones y discusiones sobre los problemas de búsqueda e información en las estadísticas perinatales tanto en paises desarrollados como en los en desarrollo. Fueron acordadas las siguientes conclusiones y recomendaciones, las que también adoptó el Comité Permanente de FIGO sobre Mortalidad y Morbilidad Perinatal.

1. El Comité reconoció la importancia básica de monitorizar la mortalidad perinatal para mejorar las normas en los cuidados perinatales. Actualmente existe no sólo una generalizada falta de datos sino también una invariable ausencia de uniformidad, tanto en la recolección como en su compilación.

2. Este tema ha merecido la mayor atención de parte de la Organización Mundial de la Salud por muchos años. También se ocuparon activamente otras organizaciones internacionales, como: La Federación Internacional para la Salud Familiar (IFFH), el Programa Internacional para Investigaciones en Fertilidad (IFFH), (actualmente conocido como Salud Familiar Internaciones (FHI)) y la Asociación Internacional para la Salud Materna y Neonatal (IAMANEH). A nivel nacional también han contribuido algunas instituciones oficiales y privadas. Sin embargo, el progreso ha sido minimo, en general, y FIGO deberá urgir a sus sociedades miembros para que concentren sus esfuerzos en estas actividades.

3. Existe una gran disparidad de criterios en lo relativo al volumen y carácter del problema tanto entre como dentro de los diferentes paises. Crean estas disparidad distintos factores como la geografia, las condiciones socioeconómicas y educacionales, el nivel de mortalidad perinatal y la infraestructura del pais, incluyendo las posibilidades do los servicios de salud.

4. Para satisfacer las diversas necesidades el Comité recomienda a FIGO solicitar a las sociedades miembros que organicen comités de mortalidad perinatal en todos los niveles. Una primera función de estos comités debería ser la motivación y entrenamiento del personal sanitario dedicado a la recolección, procesamiento y difusión de datos.

5. Estandarizar y uniformar las definiciones y la metodología es indispensable. La OMS ya ha publicado definiciones y recomendaciones en este terreno (ver Apéndice 1: Definiciones y Recomendaciones; Ap. 2: Mortalidad Perinatal) que han sido adoptadas por FIGO. E1 Comité apoya ampliamente su empleo. En particular llama la atención sobre el siguiente extracto (ver Ap. 1):

\section{Estadisticas de mortalidad perinatal:}

"Es recomendable que las estadisticas nacionales perinatales incluyan todos los fetos y niños nacidos con un peso de al menos 500 gramos (ó, 
cuando el peso no es determinable, la correspondiente edad gestacional (22 semanas) o el largo corporal ( $25 \mathrm{cms}$ ), en todo caso, vivos ó muertos. Es sabido que los requerimientos legales en muchos paises pueden sustentar diferentes criterios a los fines de los registros, pero se confía que los paises podrán acomodar sus procedimientos de registro e información de tal manera que los elementos requeridos para su inclusión en las estadísticas puedan ser fácilmente identificados. Se recomienda además que fetos menos manduros y los niños sean excluidos de las estadísticas perinatales salvo que existan razones legales ó válidas en contrario.

Se recomienda luego que las estadísticas nacionales incluyan fetos y niños cuyo peso esté entre los 500 y los 1000 gr. tanto por su valor inherente, como porque su inclusión facilita la integración de los informes para 1000 gr. ó más. La inclusión de este grupo de nacimientos muy inmaduros, sin embargo, dificulta las comparaciones internacionales por la diferencia existente entre países en lo relativo a sus sistemas de registro. Otro factor que afecta las comparaciones internacionales es que todos los niños nacidos vivos, independientemente de su peso, son incluidos en los cálculos estadisticos, en cambio algún límite inferior de madurez se aplica a los niños nacidos muertos.

Procurando eleiminar esos factores, se recomienda que los paises deban presentar, sólo para las comparaciones internacionales 'estadísticas perinatales estandard' dónde tanto numerador como denominador de todas las cifras sean limitados a fetos y niños cuyo peso sea de 1000 gr. o más (ó, cuándo el peso no es determinable, la correspondiente edad gestacional (28 semanas) o longitud corporal $(35 \mathrm{cms})$ )".

6. En 1979, la OMS recomendó el uso de sus certificados de causa de muerte perinatal (Ap. 2.). Pero con la finalidad de computar estadísticas de mortalidad y evaluar otras informaciones relevantes, es necesario obtener la correspondiente información de todos los nacimientos. En consecuencia, el Comité recomienda el uso universal de un certificado de nacimiento que contenga información comparable.

7. Con la progresiva mejora en la salud perinatal y en los cuidados perinatales y la reducción en el número do muertes previsibles en esta época de la vida, es inevitable que en la mortalidad residual se verá incluida una proporción creciente de niños con severas malformaciones. Estos niños podrán no tener la capacidad de conservar una vida independiente. En algunos centros, en los paises más desarrollados, las malformaciones severas son actualmente responsables del $30 \%$ al $40 \%$ de la mortalidad perinatal y esta proporción posiblemente continuará aumentando. Si usamos las estadísticas de mortalidad perinatal para evaluar la eficacia de los cuidados perinatales es importante poder separar estos niños de aquéllos que han nacido sin una malformación letal. Esta separación es también deseable cuando las estadísticas de mortalidad perinatal son empleadas para calificar y comparar los cuidados perinatales en diferentes paises, ya que la incidencia de malformaciones letales varía mucho de una región a otra. Una definición de trabajo para 
malformación letal es: una anomalia congénita (ver Clasificación Internacional de Enfermedades, 9a. Ed. Capitulo 14) que se considera como la causa principal de muerte durante el periodo fetal y neonatal.

8.1. A causa de las disparidades mencionadas en el párrafo 3, son necesarios distintos niveles dentro de la complejidad de la certificación médica de nacimiento y muerte. El primer nivel deberá incluir los siguientes datos:

\section{CERTIFICACION DE NACIDOS VIVOS, MORTINATOS Y MUERTE NEONATAL}

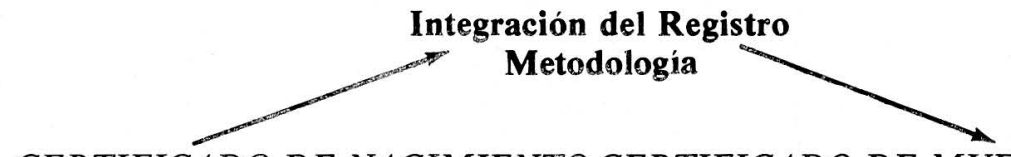

CERTIFICADO DE NACIMIENTO CERTIFICADO DE MUERTE

PERINATAL

Nombre/domicilio maternos

Nombre/domicilio maternos

Fecha/hora de nacimiento

Fecha/hora de nacimiento

Lugar del nacimiento

Nacimiento único/múltiple

Sexo del niño

Peso al nacer

Lugar del nacimiento

Nacimiento único/múltiple

Sexo del niño

Peso al nacer

Nacido vivo/muerto

Nacido/muerto

Si mortinato:

Muerto antes del parto/durante/

se ignora el momento

Necrópsia: $\mathrm{Si} / \mathrm{No}$

Se observó malformación letal:

$\mathrm{Si} / \mathrm{No}$

Si muerte neonatal:

Fecha/hora de muerte

Necrópsia: Si/No

Se observó malformación letal:

$\mathrm{Si} / \mathrm{No}$

Estos items de información reflejan a grandes rasgos los contenidos en la primera parte del Certificado de Causa de Muerte Perinatal propuesto por la OMS. (ver pág, siguiente, casilla superior).

Se ha mostrado muy útil el empleo de diferentes colores para los certificados de nacimiento y muerte, para hacer más fácil su identificación. 


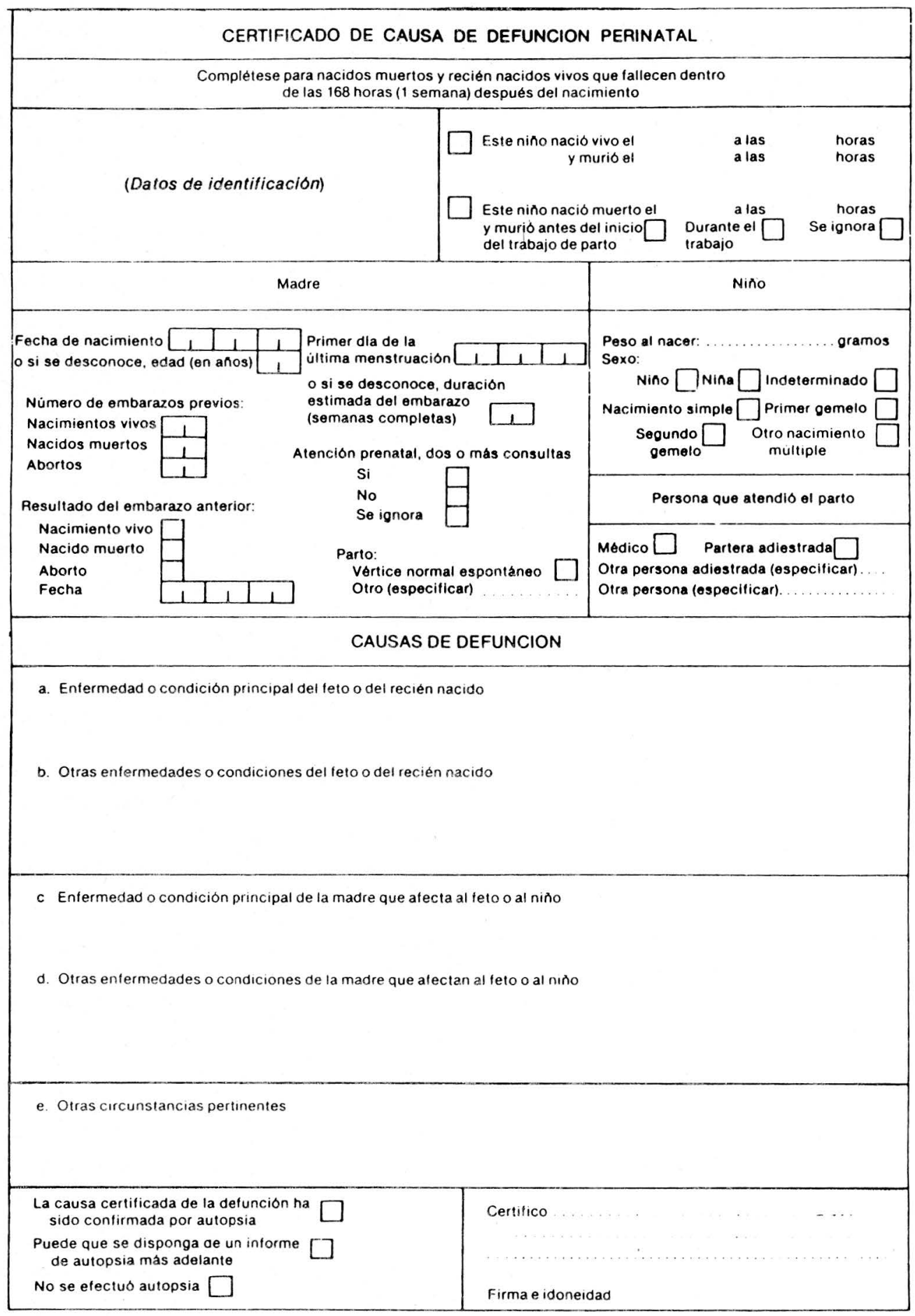


8.2. El segundo nivel, en la lista de datos para todos los nacimientos, deberá incluir además la restante información del Certificado de Causa de Muerte Perinatal de la OMS, en la siguiente forma:

Edad materna

Número de gestaciones previas

Terminación del último embarazo

Meses desde la terminación del último embarazo

Cuidados antenatales, número de visitas

Edad gestacional

Presentación en el parto

Método de parto

Atención en el parto

Para muertes perinatales:

Causas de la muerte (ver OMS formulario)

8.3. En niveles más avanzados se llenarán datos adicionales considerados relevantes para propósitos y situaciones específicas, tal como:

Educación materna

Talla materna

Cigarrillo durante la gestación

Complicaciones del embarazo

Trabajo y parto

Tipo de cuidados antenatales

Ejemplos, elegidos arbitrariamente, se encontrarán incluidos en los Ap. 3,4 y 5 .

9. No es necesario recalcar la importancia que tiene obtener la confirmación por necrópsia de las supuestas causas de la muerte.

10. Cuando sea posible, la intención será recolectar datos básicos de población. La recolección de datos básicos de la institución es también valiosa y será estimulada.

11. Va más allá del alcance de este informe discutir en detalle el análisis e información de los datos recolectados sobre mortalidad perinatal. Sin embargo, un formulario básico para tabulación se ha ilustrado en la página siguiente (MUTCH y colaboradores, 1981)

12. Sería deseable poder tener Comités de Mortalidad y Morbilidad Perinatal a nivel nacional, regional y local. Son apropiados para la integración de los mismos representantes de las siguientes disciplinas:

Obstetras

Pediatras

Patólogos

Administradores

Parteras/Nurses/Visitadoras Sociales

Epidemiólogos 
13. Para asegurar un buen resultado operacional del sistema, es esencial que exista una pronta asimilación (feedback) de los datos procesados en las estadisticas perinatales. Este feedback mantendrá el interés de todos y asegura la evaluación periódica orientando las modificaciones apropiadas para mejorar los cuidados de madres y niños.

14. Además de la mortalidad perinatal, en el futurò habrá una creciente necesidad de registrar y evaluar la morbilidad perinatal. Desgraciadamente el valor predictivo de los instrumentos disponibles al presente aún requiere evaluación. Sin embargo, información en grupos específicos del peso al nacer relacionado con algunos factores como el indice de Apgar, $\mathrm{pH}$ sanguineo de la arteria umbilical y la conducta anormal de funciones neurológicas del niño (como las convulsiones y la incapacidad para succionar), constituyen información deseable.

\section{Recomendaciones propuestas por FIGO \\ Estadísticas Perinatales para una Población Especifica}

\section{Información Requerida}

Número total de nacimientos de más de 500 gr $\ldots \ldots \ldots \ldots \ldots \ldots \ldots$

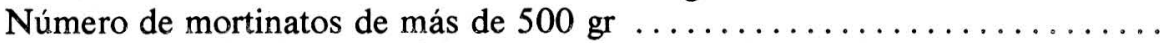

Número de muertes neonatales precoces (primera semana) $\ldots \ldots \ldots \ldots \ldots$

Número de muertes neonatales tardías $(2-4$ semanas) $\ldots \ldots \ldots \ldots \ldots \ldots$

Número de nacimientos menores de $1 \mathrm{Kg} \ldots \ldots \ldots \ldots \ldots \ldots \ldots \ldots \ldots$

Número de mortinatos menores de $1 \mathrm{Kg}$

Número de muertes neonatales precoces, de menos de $1 \mathrm{Kg} \ldots \ldots \ldots \ldots$.

Número de muertes neonatales tardias, de menos de $1 \mathrm{Kg} \ldots \ldots \ldots \ldots \ldots$.

Número de niños con malformaciones letales $\ldots \ldots \ldots \ldots \ldots \ldots \ldots \ldots$

Número de mortinatos con malformaciones letales

Número de muertes neonatales precoces con malformaciones letales .......

Número de muertes neonatales tardias con malformaciones letales...

\section{Estadísticas Perinatales:}

Cantidad de malformaciones letales por 1000 nacimientos ............

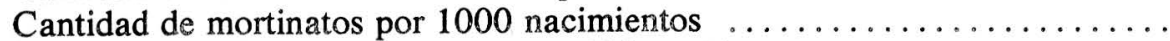
Cantidad de muertes neonatales por 1000 nacidos vivos .............. Cantidad de muertes perinatales por 1000 nacimientos

Excluyendo Malformaciones Letales (incluidos menores de $1 \mathrm{Kg}$.)

Cantidad de mortinatos por 1000 nacimientos

Cantidad de muertes neonatales por 1000 nacidos vivos

Cantidad de muertes perinatales por 1000 nacimientos 
Excluyendo nacimientos de menos de $1 \mathbf{~ K g}$ (incluidas malformacion)

Cantidad de mortinatos por 1000 nacimientos $\ldots \ldots \ldots \ldots \ldots \ldots \ldots \ldots$

Cantidad de muertes neonatales por 1000 nacidos vivos $\ldots \ldots \ldots \ldots \ldots$

Mortalidad Perinatal (cantidad) por 1000 nacimientos

15. Las estadísticas más recientes de mortalidad perinatal publicadas por la OMS pueden encontrarse en (Apps. 6 y 7.)

\section{References}

Foster, $F$. $H$. Trends in Perinatal Mortality.

World Health Statistics, 34, 138-146, 1981 (Appendix 7)

801 Maternity Care Monitoring Record or Register. International Federation for Family Health, Research Triangle Park, North Carolina 27709, USA (Appendix 4)

Medical Birth Registration in 1979 and 1980. Statistics of the National Board of Health and Welfare.

Published by the National Central Bureau of Statistics S-11581 Stockholm, Sweden 1982 (Appendix 5)

Mutch, L. M. M., Brown, N. J. Speidel, B. D., Dunn, P. M.:

Perinatal mortality and neonatal survival in Avon: 1976-9 Brit. Med. J. 282, 119, 1982

Selbmann, H. K., Warncke, W., Eissner, H. J.: Comparison of Hospitals Supporting Quality Assurance.

Methods of Information in Medicine 21, (1982), in press (Bavarian Study, Appendix 3)

World Health Organization. Report on Social and Biological Effects on Perinatal Mortality.

Geneva, 1978, Vol. I and II.

World Health Organization. Definitions and Recommendations. International Classification of Diseases, 9th Edition, Geneva, 1979, Vol I pp. 763-768 (Appendix 1)

World Health Organization: Perinatal Mortality.

International Classification of Diseases, 9th Edition, Geneva, 1979, Vol. I: pp. 731-737 (Appendix 2)

World Health Statistics Annual, 1981:

Late Fetal, Infant, Neonatal, Post-Neonatal and Perinatal Mortality. pp. 1720 (Appendix 6) 